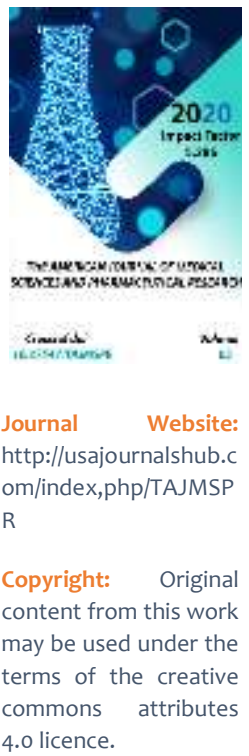

\title{
Reproductive Health And Contraceptive Behaviour Of Women Who Have Undergone Caesarean Sections
}

\author{
Nasirova Zebiniso Azizovna \\ Samarkand State Medical Institute, Uzbekistan \\ Agababyan Larisa Rubenovna \\ Samarkand State Medical Institute, Uzbekistan
}

\section{ABSTRACT}

The article contains the results of a questionnaire survey, reflecting social and hygienic characteristics of women who have undergone cesarean. cross-section (main group) and those who gave birth on their own (control group).A data analysis has also been carried out to judge attitudes to different aspects of contraception to the existing stereotype contraceptive behaviour, identify motives for women's insensitivity to modern methods for preventing unwanted pregnancies.

\section{KEYWORDS}

Caesarean section, reproductive behaviour, postpartum contraception, intergenerational interval, formal abortion, intrauterine devices.

\section{INTRODUCTION}

The high frequency of abdominal delivery is a distinctive feature of modern obstetrics. Most often, a C-section is performed on the sum of relative indications, often in the interests of the intrauterine patient 5.24. At present, the frequency of this operation is steadily increasing. In Uzbekistan, it has more than doubled over the past 10-15 years. 
Despite the close attention paid to the problem postnatal contraception, many issues related to the use of modern contraception after caesarean section remain controversial 4,7,15.

All of the above determines the need to study

Reproductive behaviour of women after caesarean sections and optimisation of the use of highly effective contraceptive methods in women with uterine scarring.

Aim of research: to improve the use of highly effective contraceptive methods in women with scars on the uterus. To study the features of reproductive behavior and awareness of contraceptive methods in women after Csection.

In order to study the peculiarities of reproductive behavior and information about contraceptive methods for women who have undergone a C-section, taking into account their social and hygienic characteristics, 500 women were surveyed. Samarkand: 320 women who had a Caesarean section in the lower uterine segment (main group) and 180 women who gave birth independently through natural birth routes (control group). In selecting the control group, the aim was to ensure that this group does not differ from the main group in a number of important social and hygienic parameters. The statistical processing was carried out using the Excel 7.0 statistical application utility. The research method was a retrospective analysis using a specially designed questionnaire in Google.

\section{RESULTS}

The age of respondents ranged from 19 to 41 years, with an average age of $26.9 \pm 1.2$ years and $27.3 \pm 1.4$ years in the main and control groups respectively. The most important factor in maintaining reproductive health women who have undergone a C-section is the solution to the problem

Unwanted pregnancy and adherence to the intergenerational interval. Artificial abortion is the most severe psychological and hormonal stress leading to functional and organic disorders in the reproductive system 5.8. The use of contraception is therefore seen as one of the most important areas in the rehabilitation of women after surgical delivery. Among the respondents in both groups, the most important reproductive age was 18-34 years ( $89.7 \%$ and $96.1 \%$ respectively). In terms of marital status, both groups of respondents were identical ( $p>0.05$ ). Married were 309 (96.6\%) women in the main group and 176 (97.8\%) women in the control group respectively. 11 (3.4\%) women in the main group and $4(2.2 \%)$ women in the control group were not married.

Distribution of respondents according to the duration of the marriage.

At the time of this survey, $99.4 \%$ of women in the main group had children (2 respondents had stillbirths in their anamnesis) and $100 \%$ in the control group. 64 (20\%) women who had had a C-section and 79 (44\%) women who had given birth on their own had one child in the family, while two 157 (49\%) and 65 (36\%) respectively. A child under 12 months of age had $28.2 \%$ of the main and $30.0 \%$ of women in the control group, and from 1 to 3 years of age $49.3 \%$ and $42.0 \%$ respectively. At the time of the survey, the average interval after childbirth in the main group was $2.2 \pm 1.7$ years and in the control group $2.5 \pm 1.8$ years. The number of women who underwent abdominal delivery 5 years or more ago was insignificant - 19 (5.9\%) and $4(2 \%)$ respondents respectively.

The distribution of respondents in the main and control groups did not have significant 
differences in age, parity and length of time between births ( $p>0.05$ ), which made it possible to consider them homogeneous by these parameters and make appropriate comparisons. Realistically, 144 (45\%) women who have undergone a Caesarean section and 243 (76\%) women who gave birth on their own ( $p>0.05$ ) plan to have a second pregnancy and delivery. However, $69.3 \%$ of the main group and $70.0 \%$ of the control group respondents want to delay a repeat pregnancy for the next three years after the actual birth and need a reliable contraceptive. Women over 34 years of age and with two or more children predominate among those who have completed reproductive functions in the main group.

A review of lactation function revealed a significantly shorter duration of lactation in the main group compared to the control group.

The choice of contraceptive methods is closely linked to women's assessment of the possibility of their negative impact on the body. In a study of 40 (12.5\%) women in the main group and 14 (7.8\%) in the control group, contraceptive use is considered to have a negative impact on health $(p>0.05)$. Negative use of hormonal contraception by $51.0 \%$ of the women in the main group and $48.0 \%$ of the control group attributed it to weight gain and metabolic effects ( $p>0.05)$. Negative use of intrauterine devices by $16.7 \%$ of women after Caesarean section due to possible scar damage and $14.0 \%$ of women who gave birth independently due to increased menstrual blood loss and the possibility of inflammatory diseases.

According to our data, the main role in the decision to use contraception is played by the spouse/spouse (41.9\% and $37.8 \%$ respectively). Only $10.9 \%$ of women in the main group and $20 \%$ in the control group made an independent decision on the use of contraception, and only $19.1 \%$ of women in the main group and $16.1 \%$ of women in the control group consulted with health professionals. The survey data shows that only $11-24 \%$ of women in both groups received information on contraception from health workers. Of particular note is the lack of regular information in the media. $20 \%$ of women in the main group and $17.8 \%$ of women in the control group who received information about contraceptive methods were patients who turned to health workers for contraceptives themselves. According to our study of active primary health care counselling on contraception methods, there are no women who have undergone caesarean sections, just like those who gave birth on their own.

\section{DISCUSSION}

Postpartum family planning is an important component of maintaining women's health, reducing the risk of unwanted pregnancies and increasing the birth interval $[2,6,23]$.

Despite the fact that WHO experts have formulated medical criteria for the acceptability of contraceptive methods in women after childbirth and after cesarean section [24], many questions of this problem remain unresolved. In particular, according to the findings of a recent research, the majority of women do not use effective methods of contraception during a year after cesarean section, regardless of marital and social status.

Our studies confirm data of several authors about the necessity for recommendations of highly effective, long-term reversible methods of contraception to patients after childbirth in order to preserve their somatic and reproductive health [18]. At the same time, we absolutely agree with the opinion of Haider $\mathrm{S}$ et authors (2020) on the need for qualified 
counseling of patients on contraception. Only highly motivated and informed women will use modern methods of contraception, which is also indicated in studies of Harney $C$ et authors, 2014. This promotes an informed choice, longterm usage and the absence of an adverse effect of contraception on the psychological status of women. Indeed, the choice of methods of contraception depends on the woman's reproductive plans, the number of children in a family and the cost of contraceptions. In our country, coppercontaining IUCs are provided free of charge. A large retrospective study by Howard B et al in 2014 indicates the importance of number of children in a family and the availability of insurance payment when the patient decides to choose highly effective reversible long-term contraception.

According to our data, after childbirth and after cesarean section, most patients prefer intrauterine contraception to all other modern highly effective methods of contraception. Our earlier study showed that if the necessary conditions are met, intraoperative IUC insertion can be absolutely safe [6]. However, the data from present survey showed that most women after cesarean section wish to delay the introduction of the IUC, for example implementing not in the post-placental period and not during the cesarean section, but after an onset of next menstruation. This appears to be due to insufficient prenatal counseling. As the survey shows, insufficient counseling is associated with many factors: lack of sufficient time and desire among medical workers, insufficiently trained medical personnel, the presence of urban myths and misunderstandings about the adverse effects of modern contraceptives on a body of the nursing mother.
And in general, immediately after childbirth or before a cesarean section, most patients are worried about other issues, such as the state of their own health and the health of newborn, the sufficiency of lactation, attention to them from a family and so on. According to literature, if there is a possibility of a postpartum visit, delayed IUC insertion is safer and less expensive (Starr KA et al, 2015), since later, during the first month after childbirth, with a recovery of sexual relations, women begin to worry about unwanted pregnancy. At this time, to increase the adherence to usage of modern methods of contraception, it may be beneficial to conduct not only visits to the doctor in the postpartum period, but also visits of nurses, pediatricians, and general practitioners to patients' home. At the same time, Zaconeta A. M et al (2019) recommends that in countries with a high level of caesarean section, the IUC should be inserted intraoperatively without prenatal counseling. Such tactics are unacceptable in our country and, in our opinion, can lead to discrediting of the method.

\section{CONCLUSIONS}

Thus, the reproductive behavior of women who underwent a cesarean section is characterized by a positive attitude towards having children in the future and by desire to postpone a repeated pregnancy for the next three years after the operation regardless of social and hygienic parameters. But at the same time, it is also characterized by using of low-effective methods of contraception in the first year after surgery, and therefore a high frequency of induced abortions.

In this regard, special attention should be paid to the survey data on the awareness of women on contraceptive methods: no woman, both in the main and in the control group, had information about availability of 4-5 methods 
of contraception. 105 (32.8\%) women in the main group and 61 (33.9\%) women in the control group knew about 3 methods of contraception, i.e. this information in both groups did not have significant differences ( $p>$ $0.5) .160(50 \%)$ women in the main group and $86(47.8 \%)$ women in the control group $(p>0.5)$ were informed about only two methods of contraception. The remaining 55 (17.2\%) and 33 (18.3\%) ( $p>0.5$ ) of both groups, respectively, knew about just 1 method of preventing unwanted pregnancy. $83.3 \%$ of women in the main group and $90 \%$ of women in the control group knew about the IUC, but at the same time they believed that postpartum insertion could adversely affect their health, and preferred not to use this method until an onset of first menstruation. It should be especially noted that none of the respondents answered about possibility of using the method of lactational amenorrhea - all 100\% of women knew that breastfeeding can protect against pregnancy, but no one pointed out the 3 main conditions of MLA - exclusive breastfeeding, the child's age is less than 6 months and absence of menstruation.

\section{CONCLUSION}

1. The majority of patients after cesarean section wish to postpone a birth of their next child for 2-3 years, but at the same time, they do not have reliable information about the possibility of using modern methods of contraception in the antenatal period.

2. In order to increase the patient's adherence to the widespread use of contraceptive methods, it is necessary to improve active counseling among pregnant women, women in labor and parturient women with the involvement of not only maternity hospital staff, but also primary health caregivers, as well as the media.

3. For women who have undergone cesarean section, the use of modern highly effective methods of contraception will not only guarantee both the preservation of the intergenetic interval and prevention of abortion.

\section{REFERENCES}

1. Advances in contraception: new options for postpartum women Taub $\mathrm{RL}$ and Jensen JT. Expert Opin Pharmacother 2017 - Review.

2. Barbosa RM, et al. Differences in the Access to Sterilization between Women Living and Not Living with HIV: Results from the GENIH Study, Brazil PLoS One 2016.

3. Basendowah M., Alabdulqader M., Alabdulqader O., Hakami M. Cureus. 2020 12(1): c6644. Doi: 10,7759/ cureus.6644

4. Borges A. L. V., dos Santos O. A., Fujimori E. Concordance between intention to use and current use of contraceptives among six-month postpartum women in Brazil: The role of unplanned pregnancy //Midwifery. 2018. - T. 56. - C. 94-101.

5. Brunson M. R. et al. Postpartum contraception: initiation and effectiveness in a large universal healthcare system //American journal of obstetrics and gynecology. - 2017. T. 217. - №. 1. - C. 55. e1-55. e9.

6. Daniele MAS, et al. Provider and lay perspectives on intra-uterine contraception: a global review. Reprod Health 2017 - Review.

7. Diedrich JT, et al. Long-acting reversible contraception in 
adolescents: a systematic review and meta-analysis Am J Obstet Gynecol 2017 - Review.

8. Garcia G. et al. Trends and disparities in postpartum sterilization after cesarean section, 2000 through 2008 //Women's Health Issues. - 2015. - T. 25. - №. 6. c. 634-640.

9. Goldthwaite $L M$ and Shaw KA. Immediate postpartum provision of long-acting reversible contraception Curr Opin Obstet Gynecol 2015 Review.

10. Goldthwaite LM, et al. Postpartum intrauterine devices: clinical and programmatic review Am J Obstet Gynecol 2018 - Review.

11. Guidelines Fleming $\mathrm{N}$, et al. J Adolescent Pregnancy Obstet Gynaecol Can 2015.

12. Haider S. et al. A Novel Approach to Postpartum Contraception Provision Combined with Infant Care: A Randomized, Controlled Trial //Women's Health Issues. - 2020. - T. 30. - №. 2. - C. 83-92.

13. Hormonal contraception and breast cancer, what more do we need to know? Marsden J. Post Reprod Health 2017 - Review.

14. Hubacher D, et al. Long-acting reversible contraceptive acceptability and unintended pregnancy among women presenting for short-acting methods: a randomized patient preference trial Am J Obstet Gynecol 2017 - Clinical Trial.

15. Kumar S. et al. Women's experience with postpartum intrauterine contraceptive device use in India //Reproductive health. - 2014. - T. 11. №. 1. - C. 32.
16. Makins A., Cameron S. Post Pregnancy Contraception //Best Practice \& Research Clinical Obstetrics \& Gynaecology. - 2020.

17. MacDonald EJ, et al. Contraception post severe maternal morbidity: a retrospective audit Contraception 2015.

18. Mishra S. Evaluation of safety, efficacy, and expulsion of post-placental and intra-cesarean insertion of intrauterine contraceptive devices (PPIUCD) //The Journal of Obstetrics and Gynecology of India. - 2014. - T. 64. - №. 5. - C. 337343.

19. Raine $T R$, Foster-Rosales $A$, Upadhyay UD, Boyer CB, Brown BA, Sokoloff A, et al. One-year contraceptive continuation and pregnancy in adolescent girls and women initiating hormonal contraceptives. Obstet Gynecol. 2011;117:363-71.

20. Samuel E., Myers E., Morse J. IUD insertion after cesarean section: a cost decision analysis of insertion at time of delivery versus at postpartum followup //Contraception. - 2014. - T. 90. - №. 3. - C. 327-328.

21. Shanavas A., Jacob S., Chelamma N. Outcome of immediate postpartum intrauterine contraceptive device in caesarean versus vaginal insertion: a comparative study //International Journal of Reproduction, Contraception, Obstetrics and Gynecology. - 2017. - T. 6. - №. 2. - C. 695.

22. Starr K. A. et al. Postpartum contraception use by urban/rural status: an analysis of the Michigan pregnancy risk assessment monitoring system data //Women's Health Issues. 2015. - T. 25. - №. 6. - C. 622-627. 
23. Wilkie G. L. et al. Effects of obstetric complications on adolescent postpartum contraception and rapid repeat pregnancy //Journal of pediatric and adolescent gynecology. - 2016. - T. 29. - №. 6. - C. 612-616.

24. World Health Organization. Medical eligibility criteria for contraceptive use. 4th ed. Geneva: WHO; 2009. Available at: http://www.who.int/reproductivehealth/publications/mec/family_planni ng/9789241563888/en/index.html

25. Zaconeta A. M. et al. Intrauterine Device Insertion during Cesarean Section in Women without Prenatal Contraception Counseling: Lessons from a Country with High Cesarean Rates //Revista Brasileira de Ginecologia e Obstetrícia/RBGO Gynecology and Obstetrics. - 2019. - T. 41. - №. 08. - C. 485-492. 